\title{
Sigmund Kvam*
}

\section{Translatorische Relevanz und Übersetzungsstrategie. Eine Fallstudie zur Übersetzungsdidaktik}

\section{Einleitung}

Wer authentische Übersetzungsfälle analysiert hat, wird ziemlich schnell zweierlei feststellen müssen: Erstens könnte der Zieltext hinsichtlich Inhalt und Struktur vom Ausgangstext z.T. deutlich abweichen. Zweitens lässt sich nachweisen, dass ein Ausgangstext in einer gegebenen Zielsprache unterschiedliche, sehr stark von einander abweichende Zieltexte aufweisen kann, ohne dass zwischen diesen notwendigerweise nennenswerte Qualitätsunterschiede vorliegen. Der Zieltext ist also in vielen authentischen Übersetzungsfällen alles andere als eine Kopie des Ausgangstexts und ein und derselbe Ausgangstext kann offensichtlich in viele verschiedene Richtungen übersetzt werden. Hierzu einige Beispiele:

Bei der Übersetzung eines Informationsfaltblatts der Mittelalterkirche Berg kirke in Halden in Norwegen ins Deutsche wurden nach Rücksprache mit dem Auftraggeber Sachverhalte geändert. Für die Zielgruppe, deutschsprachige Touristen in Halden, war der Hinweis im Ausgangstext auf Veranstaltungen im Gemeindehaus irrelevant, da diese erstens in norwegischer Sprache, zweitens nicht im Sommer stattfinden. Hinzugefügt wurde jedoch Information über eine Übersetzung der Liturgie der norwegischen Kirche ins Deutsche. Aktualisiert bzw. korrigiert wurden Telefonnummern sowie auch einige Sachfehler im Ausgangstext (z.B. romersk (römisch) statt romansk (romanisch) zur Bezeichnung von Elementen des Baustils der Kirche).

\footnotetext{
* Sigmund Kvam

Høgskolen i Østfeld

Os Allé 5

$N$-1757 Helden

Sigmund.kvam@hiof.no
} 
In einer Analyse zur Sententialität und Nominalität als Übersetzungsstrategien in norwegischen Übersetzungen deutscher Sachprosatexte (Solfjed 2000) werden Fälle besprochen, in denen „die Argumentation im Translat weniger klar als im Original vermittelt wird“ (ebd., 298): Um einen textsortengerechten Stil in den norwegischen Zieltexten zu erreichen, wird die in einem Satz enthaltene Information im deutschen Ausgangstext im norwegischen Zieltext oft auf mehrere Sätze verteilt. Dabei wäre oft eine leichte Modifizierung des Inhalts des Ausgangstextes zugunsten eines textsortengerechten Stils im Zieltext in Kauf zu nehmen ${ }^{1}$.

In Berger/Nord 2000 wird gezeigt, dass ein und derselbe Ausgangstext - das Neue Testament - in unterschiedlichen Übersetzungskontexten in ein und dieselbe Sprache - das heutige Deutsch - sehr unterschiedlich übersetzt worden ist. Dabei wäre es völlig unberechtigt zu behaupten, die eine Übersetzung sei falsch und die andere etwa richtig. Denn es handelt sich hier um den empirischen Nachweis von zwei grundsätzlich verschiedenen Übersetzungsaufträgen: Die eine Übersetzung, das Münchener Neue Testament, ist eine wortgetreue Übersetzung ins Deutsche, um die sprachlichen Strukturen des griechischen Ausgangstextes möglichst genau abzubilden, während die Lutherbibel in tradierter, aber immerhin moderner sprachlicher Gestaltung die Verständlichkeit der Bibel für den modernen Menschen bezweckt.

Durch diese Beispiele wird ersichtlich, dass die Übersetzung eines gegebenen Textes durch Merkmale des Ausgangstextes nicht allein bestimmt wird ${ }^{2}$, sondern vor allem auch durch von Übersetzungssitua-

\footnotetext{
1 Vgl. hierzu Solfjeld 2000, 296ff. sowie eine Diskussion solcher Textbildungsstrategien in Kvam 2002, 132ff..

2 Es würde hier zu weit führen, auf die jahrelangen Kontroversen zwischen einem retrospektiven, am Ausgangtext orientierten und durch Äquivalenz definierten und einem prospektiven, am Zieltext orientierten und durch Skopos bzw. Adäquatheit definierten Übersetzungsbegriff systematisch einzugehen. Nichtsdestotrotz dürfte klar sein, dass Übersetzen grundsätzlich sowohl prospektiv als auch retrospektiv ist. Eine Ballade wie Schillers Die Bürgschaft wird im europäischen Kulturkreis nur unter Beibehaltung der lyrischen Form als Übersetzung akzeptiert werden. In anderen Textsorten, wie im besprochenen Übersetzungsfall von Berg kirke in der norwegischen Stadt Halden, werden im Rahmen einer Übersetzung im Zieltext referentielle Elemente des Ausgangtextes behalten, gestrichen und neue hinzugefügt. Vgl. hier zu auch die Diskussion von translatorischer Relevanz im Verhältnis zu Adäquatheit in 2.4.
} 
tion zu Übersetzungssituation variierende spezifische Vorgaben für die jeweilige Zieltextherstellung. Bei der Evaluation einer Übersetzung müssen also zusätzlich zum Ausgangstext auch die situativen Erfordernisse im jeweiligen Übersetzungsfall berücksichtigt werden. Wie im Fall der Bibelübersetzung im Münchener Neuen Testament, kann eine rein wörtliche Übersetzung gefragt werden, sie muss es aber nicht sein, wie die Übersetzung der gleichen Textstellen in der Lutherbibel zeigt. In den anderen Übersetzungsbeispielen werden durch kommunikative Forderungen an den zu produzierenden Zieltext referentielle - wie im Fall der Übersetzung des Faltblattes für Berg kirke - und textstrukturelle zugunsten inhaltlicher - wie im Fall der analysierten Sachprosatexte in Solfjeld 2000 - Aspekte des Ausgangstextes im Zieltext deutlich geändert.

Die unterschiedliche Gewichtung von verschiedenen textuellen Aspekten des Ausgangstexts bei der Zieltextproduktion ist nicht nur theoretisch, sondern auch in übersetzungsstrategischer und -didaktischer Hinsicht interessant: Studierende tun sich nicht nur schwer, geeignete Strategien für eine im jeweiligen Übersetzungsfall angemessene Gewichtung von textuellen Aspekten des Ausgangstexts auszuarbeiten. Vielen Studierenden fällt es auch schwer, die Frage nach einer unterschiedlichen Gewichtung von Ausgangstextinhalten überhaupt zu stellen.

In der vorliegenden Arbeit möchten wir zunächst auf der Basis eines pragmatischen Bedeutungs- und Textbegriffs das Problem der unterschiedlichen Gewichtung von Ausgangstextmerkmalen über den noch näher zu definierenden Terminus translatorische Relevanz theoretisch begründen (2). Anschließend wollen wir durch eine kleine Fallanalyse von Prüfungsklausuren den konkreten Umgang mit translatorischer Relevanz in der Translationsstrategie von Studierenden analysieren (3). Zum Schluss werden übersetzungsdidaktische und übersetzungsstrategische Implikationen von translatorischer Relevanz skizziert (4). 


\section{Zum theoretischen Status von translatorischer Relevanz}

\subsection{Vorbemerkung}

Durch die oben zitierten Beispiele wird ersichtlich, dass Übersetzen in bezug auf eine Bindung zum Ausgangstext multidimensional ist. Dies hat zentrale theoretische Folgen für die linguistischen Grundlagen einer Übersetzungswissenschaft. Es würde jedoch hier zu weit führen, auf eine ausführliche Diskussion der linguistischen Grundlagen von Übersetzen einzugehen. Erwähnen möchten wir trotzdem zwei wichtige linguistische Aspekte, und zwar Bedeutung und Text.

\subsection{Zum Bedeutungsbegriff}

Die meisten sprachlich-kommunikativen Zeichen sind bekanntlich symbolisch und bedeuten als Ansammlung von Lauten oder Schriftzeichen an sich ,gar nichts'. Erst durch die Verwendung von Menschen in einer Kommunikationssituation wird ihnen Bedeutung zugeschrieben, indem die an der Kommunikation Beteiligten durch die Verwendung dieser Zeichen Bedeutung gemeinsam herstellen 3 . Bei jeder Textproduktion findet also eine neue Bedeutungskonstitution statt. Beim Übersetzen ist das nicht anders. Auch hier wird von den Beteiligten Bedeutung konstituiert, allerdings in der Form einer als Übersetzen konventionalisierten Textproduktion.

Der hier zugrundegelegte Bedeutungsbegriff ist also handlungsbezogen, da Bedeutung erst durch das Handeln in konkreten Kommunikationssituationen hergestellt werden kann. Denn Bedeutung kann nicht unabhängig von Situation, Konvention, von Kultur im weitesten Sinne existieren und deshalb auch nicht als ein von der Kommunikation getrenntes System beschrieben werden.

Dies hat auch zur Folge, dass Funktion im Sinne von Handlungsinteresse bzw. intendierter Wirkung von Bedeutung schwer getrennt werden kann. Denn mit der Bedeutungskonstitution werden automatisch Handlungsinteressen verfolgt, die eine Sachverhaltsdarstellung vielschichtig und nach verschiedenen Funktionsaspekten4 wesentlich be-

3 Vgl. hierzu u.a. die Erörterung der Thesen von Gumperz in Auer 1999, 164ff..

4 Vgl. hierzu u.a. Adamzik 2000, 103ff.. 
stimmen. Die Tatsache, dass Bedeutung nicht außerhalb von Kommunikation als statisches, autonomes System existiert, sondern erst in Kommunikationssituationen von den Beteiligten an Ort und Stelle selbst dynamisch konstituiert wird, ist in der gesprächsanalytischen Forschung ein längst bekanntes Faktum und wird in verschiedenen Arbeiten auf solider empirischer Grundlage nachgewiesen5. Aber auch in der geschriebenen Kommunikation kann von einer interaktiven Bedeutungskonstitution argumentiert werden. Zwar wird hier die Kommunikationssituation von den Beteiligten nicht an Ort und Stelle gleichzeitig konstituiert, sondern im Sinne von Ehlich als zeitlich und räumlich zerdehnte Kommunikation (Ehlich 1996, 18ff.) realisiert. Mit der Textproduktion sucht der Textproduzent über den Produktionsort und -zeitpunkt der kommunikativen Handlung hinaus eine im Sinne seiner Handlungsinteressen spezifische Textbedeutung beim Rezipienten zu bewirken. Dadurch antizipiert er gewissermaßen eine Textbedeutung, die schließlich im Dialog zwischen Rezipienten und Text endgültig etabliert wird.

\subsection{Zum Textbegriff}

Eine Folge des oben diskutierten pragmatischen Bedeutungsbegriffes ist eine Auffassung von Text als pragmatischer Einheit ${ }^{6}$. Das bedeutet nicht, dass traditionelle strukturelle Aspekte von Texten unwesentlich sind ganz im Gegenteil: Dass Texte grammatisch und semantisch strukturiert sind, z.B. im Sinne von Superstrukturen bzw. Macrostrukturen ${ }^{7}$ und Kohärenz/Kohäsionn ${ }^{8}$ sind längst bekannte Fakten, die selbstverständlich auch in einer pragmatisch orientierten Textlinguistik relevant sind. Aber über eine semantische und grammatische Strukturiertheit hinaus sind Texte auch strukturierte Handlungseinheiten. Die Handlungs-

\footnotetext{
5 Vgl. hierzu u.a. die Arbeiten in Nothdurft/Reitemeier/Schröder 1994, in denen Beratungsgespräche auf der Grundlage authentischer Aufnahmen analysiert werden. Ein zentraler Problembereich in allen drei Arbeiten sind eben die gemeinsame Herstellung von Bedeutung und damit verbundene Kommunikationsstrategien und -probleme.

6 Vgl. hierzu auch die Diskussion der Relevanz eines pragmatischen Textbegriffs für das Übersetzen in Engberg 2002, 159f.

7 Vgl. einführend u.a. Heinemann/Heinemann 2002, 74ff. sowie als Analyseinstrument in Tirkkonen-Condit 1985, 21ff..

8 Vgl. hierzu u.a. Halliday 1985, 287ff..
} 
strukturen von Texten sind eine logische Folge der Tatsache, dass durch Texte soziale Bedeutung konstituiert wird und durch Texte soziale Handlungsziele zu realisieren gesucht werden. Im Sinne eines solchen handlungsbezogenen Textbegriffs können wir Texte als delimitierte, konventionalisierte und strukturierte kommunikative Einheiten zur Realisierung von Handlungsinteressen definieren. Ein solcher Textbegriff ist funktional in dem Sinne, dass unter Funktion die Verfolgung von Handlungsinteressen verstanden wird. Diese sind im Text nicht so eindeutig nachweisbar wie beispielsweise formal-grammatische Phänomene, sie lassen sich jedoch interpretativ über eine Analyse von grundsätzlich multifunktionalen Handlungsschritten im Text ermitteln9. Die hier kurz skizzierten textlinguistischen Grundvoraussetzungen übersetzungswissenschaftlicher Analysen machen deutlich, dass die Linguistik eine unabdingbare Voraussetzung für die Übersetzungswissenschaft ist, wobei hier eben nicht eine Systemlinguistik im Sinne von Klein 1991, sondern eine pragmatische Textlinguistik bzw. eine Wissenschaft vom sprachlichen Verhalten wie dies in Ortner/ Sitta 2002, 5ff. gefordert wird: Da Texte zentrale Mittel sowohl der individuellen als auch der sozialen Wissenskonstitution sind, seien die Grundlagen einer Sprachwissenschaft nicht kleinste semantische Einheiten, „herausgenommen aus aller Praxis“ (ebd.,9), sondern die „Gesamtzeichen für individuelles und soziales Wissen“ (ebd.). Sprache sei demnach „als von Menschen konstituierte Verhaltensform zwischen Menschen“ (ebd.), also als Handlungsinstrument zu betrachten und eben nicht als ein abstraktes System von kommunikationsbereinigten Kategorien.

\section{3. Übersetzungsbegriff und translatorische Relevanz}

In diesem linguistischen Rahmen ist eine Übersetzung wie jeder andere Text multifunktional in dem Sinne, dass jedem Handlungsschritt nicht nur eine, sondern grundsätzlich mehrere Funktionen zugeschrieben werden können ${ }^{10}$. Über die allgemeinen Textmerkmale hinaus zeichnet sich Übersetzen zum einen als interlinguale und interkulturelle Text-

9 Vgl. hierzu die Kritik des sprechakttheoretischen Ansatzes in Adamzik 2000, $92 \mathrm{ff}$. und Analysebeispiele in Brinker 2002.

10 Vgl. u.a. Adamzik 2000, 103ff.. 
produktion aus. Zum anderen besteht Übersetzen aus zwei separaten sprachlich-kommunikativen Handlungen, die mit einander über variierende, aber konventionalisierte und deshalb regelgeleitete Intertextbeziehungen verbunden sind und von der aktuellen Diskursgemeinschaft als Übersetzen bezeichnet und akzeptiert werden. Die Tatsache, dass es sich beim Übersetzen um zwei von einander getrennte sprachlich-kommunikative Handlungen handelt, bedeutet, dass der Zieltext allein wegen unterschiedlicher Produktions- und Rezeptionsbedingungen von Ausgangstext und Zieltext keine direkte Kopie des Ausgangstextes sein kann ${ }^{11}$. Noch dazu kommt - wie bereits in 1 . erwähnt -, dass der Textproduktionsauftrag für den Zieltext von dem des Ausgangstextes abweichen kann ${ }^{12}$ bzw. dass ein und derselbe Ausgangstext im Rahmen verschiedener Textproduktionsaufträge höchst unterschiedlich übersetzt werden kann ${ }^{13}$. Daraus folgert, dass im Ausgangstext enthaltene Bedeutungselemente im Zieltext einen anderen Stellenwert haben können als im Ausgangstext. Denn der Zieltext steht in einem anderen Kultur- und Kommunikationskontext, mit neuen Handlungsbeteiligten und grundsätzlich neuen Handlungsinteressen. Vor diesem Hintergrund können wir translatorische Relevanz wie folgt definieren:

Unter translatorische Relevanz verstehen wir den im jeweiligen Übersetzungsfall variierenden Stellenwert von semantischen, strukturellen und aktionalen Aspekten des Ausgangstextes für die Handlungsinteressen des Initiators vom Zieltext.

Semantisch heißt in diesem Zusammenhang die unterschiedliche Relevanz von referentiellen Elementen im Ausgangstext für die Handlungsinteressen des Zieltextinitiators ${ }^{14}$. Strukturell heißt in diesem

\footnotetext{
11 Beim Simultandolmetschen könnte allerdings von einer Wiederholung des Ausgangsdiskurses gesprochen werden, da ja hier erstens von einer und nicht zwei Kommunikationssituationen die Rede ist und Produktion und Rezeption des Diskurses im Prinzip gleichzeitig stattfinden.

12 Vgl. hierzu homologous translation in Nord 1997, 52 und diaskopische Translation in Prunc 1997, 48f..

13 Vgl. hierzu die Unterschiede zwischen dem in Berger/Nord 2000 besprochenen Münchener Neues Testament und den neutestamentlichen Text in der Lutherbibel.

14 Ein Beispiel wäre die in 1. besprochene Übersetzung Berg kirke, in der ja Informationen über Veranstaltungen im Gemeindehaus im Zieltext gestrichen wurden, weil diese in bezug auf deutsche Touristen als Zielgruppe als nicht relevant angesehen wurden.
} 
Zusammenhang eine im Sinne der Handlungsinteressen des Zieltextinitiators spezifische Neugestaltung von strukturellen Elementen im Ausgangstext ${ }^{15}$. Alle diese unterschiedlichen Relevanzsetzungen sind aktional im Sinne der Intentionalität von kommunikativen Handlungen. Aber auch die übergeordneten Handlungsinteressen können zwischen Ausgangstext und Zieltext so deutlich variieren, dass auch eine vorwiegend aktional bedingte Relevanzsetzung angenommen werden kann ${ }^{16}$. Durch diesen unterschiedlichen Umgang mit Ausgangstextelementen bei der übersetzerischen Zieltextproduktion wird im Sinne unserer Ausführungen in 2.1. versucht, die Interpretation des intendierten Zieltextlesers in Richtung der intendierten Wirkung zu lenken, um somit die gewünschte interaktiv herzustellende Textbedeutung zu antizipieren.

\subsection{Translatorische Relevanz und Adäquatheit}

Wie in 1. erwähnt, kann im Rahmen der hier angegebenen Problemstellung auf eine generelle Diskussion übersetzungswissenschaftlicher Modelle und Grundbegriffe nicht eingegangen werden. Aber es wäre schon angebracht, die Platzierung des gerade erläuterten Begriffs der translatorischen Relevanz im Verhältnis zu zentralen Nachbarkategorien etwas genauer zu diskutieren. Dabei werden wir uns exemplarisch auf die zentrale übersetzungsprozessorientierte Kategorie Adäquatheit am Beispiel der Ausführungen in Reiß 2000 konzentrieren.

Zwischen Adäquatheit und translatorischer Relevanz liegen sowohl Gemeinsamkeiten als auch Unterschiede vor. Beiden gemeinsam ist die Handlungs- bzw. Prozessorientiertheit und die Auswahl von sprachlichen Mitteln zur Erfüllung des Zwecks bzw. der mehr oder weniger

\footnotetext{
15 Beispiele sind die in 1. erwähnten unterschiedlichen Textgestaltungsstrategien und damit zusammenhängenden unterschiedlichen syntaktischen Präferenzen im Ausgangstext und Zieltext in Solfjeld 2000.

16 Ein Beispiel wäre die in Berger/Nord 2000 analysierte Bibelübersetzung. Hier wird ein ,missionsgerechter' Ausgangstext in eine Art Studienausgabe für theologische Interessierte umgewandelt - im Sinne der Multifunktionalität von kommunikativen Handlungen zwar immer noch missionierend und so gesehen im Sinne von Nord 1997, $50 \mathrm{ff}$. instrumentell. Der Zieltext ist jedoch auch dahingehend eine lexikalische und strukturelle Kopie des Ausgangstextes, dass der Leser gewissermaßen den Ausgangstext in einer bekannten Sprache mitlesen kann und deswegen im Sinne von ebd., 47ff. auch deutlich dokumentarisch.
} 
komplexen Handlungsinteressen des Zieltextinitiators. Dies bedeutet, dass der Ausgangstext nicht ,heilig' ist und deshalb oft bewusst bearbeitet wird, „,weil der Zieltext anderen Zwecken dienen soll als der Ausgangstext“" (Reiß 2000, 107).

Unterschiedlich sind vor allem zwei Aspekte. Zum einen ist Adäquatheit ein normatives Prinzip für eine funktionsgerechte Herstellung von Übersetzungen, translatorische Relevanz dagegen von einem pragmatischen Textbegriff und einer damit verbundenen Textlinguistik abgeleitet. Bei der translatorischen Relevanz handelt es sich also um eine textlinguistische Analysekategorie und nicht um ein normatives Prinzip. Zum anderen ist Adäquatheit - eben als normatives Prinzip - generell orientiert an einer im Einklang mit dem Übersetzungsauftrag angemessenen Zieltextproduktion. Translatorische Relevanz ist zwar auch an der Produktion eines funktionsgerechten Zieltextes orientiert, aber kein allgemeines Prinzip: Hier ist dagegen von einer Textanalysekategorie die Rede, die auf einen Ausschnitt des Übersetzungsprozesses bezogen ist, und zwar auf einen Teil der Analyse des Ausgangstextes17. Bei der translatorischen Relevanz handelt es sich also um ein textlinguistisch fundiertes strategisches Werkzeug. Als solches ist sie einerseits ein Beispiel dafür, wie übersetzungsrelevante Analysekategorien auf der Grundlage einer pragmatischen Textlinguistik gewonnen werden können sowie andererseits auch ein Hinweis auf die Relevanz der Textlinguistik als theoretischer Grundlage einer Übersetzungswissenschaft.

\section{Translatorische Relevanz als didaktisches Problem}

\subsection{Vorbemerkung}

Fragen der translatorischen Relevanz sind für Studierende recht neu und z.T. verwirrend. Ohne hier in didaktische Spekulationen zu verfallen, würden wir vor dem Hintergrund langjähriger didaktischer Praxis die folgende Behauptung wagen: Studierende übersetzen in der ersten Lernphase sehr ausgangstexttreu; der Maßstab für den Ziel-

17 Die Verwendung von translatorischer Relevanz als Strategiekomponente setzt natürlich eine textlinguistisch basierte Analyse des Ausgangstextes als eigene, ausgangssprachliche kommunikative Handlung voraus. Erst auf dieser Grundlage kann der Frage nach dem Stellenwert textueller Kategorien in dem Ausgangstext für einen an den Handlungsinteressen des Initiators orientierten Zieltext nachgegangen werden. 
text ist vor allem die Abfolge der Sätze im Ausgangstext. Nach der ersten Beschäftigung mit funktionalen Aspekten vom Übersetzen, vor allem mit einer zielgruppengerechten Zieltextproduktion, wird manchmal so ,frei’ übersetzt, dass eine übersetzungsauftragsspezifische Bindung des Zieltextes zum Ausgangstext total in Vergessenheit gerät. Vor dem Hintergrund einer solchen Ausgangstextsklaverei bzw. Ausgangstextemanzipation haben wir Fragen der in 2.3. erläuterten translatorischen Relevanz bei Prüfungsklausuren von Studierenden im 4. Semester Wirtschaftsdeutsch genauer untersucht.

\subsection{Materialgrundlage und didaktisches Konzept}

Bei dieser kleinen Untersuchung handelt es sich um neun Studierende von deutscher Wirtschaftskommunikation im 4. Semester am Fachbereich Fremdsprachen und Sozialwissenschaften der Gesamthochschule Østfold (Høgskolen i Østfold) in Halden in Norwegen. Von diesen 4 Semestern hat jeder Studierende das dritte Semester als Praktikum in einem deutschen Betrieb verbracht. In den ersten beiden Semestern haben die Studierenden zwar auch Übersetzungsunterricht bekommen, allerdings wurde Übersetzen hier als didaktisches Mittel für die Einübung von grammatischer, lexikalischer und idiomatischer Korrektheit im Rahmen des Fremdsprachenerwerbs eingesetzt. Im 4. Semester also nach dem genannten Betriebspraktikum - wird dann Übersetzen im professionellen Sinne angeboten: Hier geht es vor allem um die Erstellung von funktionsgerechten Texten. Diese müssen bzw. sollten selbstverständlich auch satzgrammatisch korrekt sein, darüber hinaus sind hier Fragen des textsortengerechten Stils, einer zweckorientierten Sprache - sowie die oben definierten Fragen der Prioritätensetzung beim Übersetzen, also die translatorische Relevanz, zentral. Dementsprechend unterscheidet sich die Prüfungsform von der Prüfung im 2. Semester. Während Übersetzen im 2. Semester im Rahmen einer Klausur von 5 Stunden 18 ohne Hilfsmittel und auch ohne einen spezifischen Übersetzungsauftrag geprüft wird, ist die Prüfung im 4. Semester ganz anders. Die Studierenden haben für zwei spezifische Übersetzungsaufträge - einen vom Deutschen ins Norwegische und einen

18 Zu dieser Prüfungsklausur gehören auch das Verfassen von zwei Geschäftsbriefen und grammatische Fragen. 
vom Norwegischen ins Deutsche - sowie flankierende Fragen zur Textanalyse und Übersetzungsstrategie - 27 Stunden zur Verfügung19. In bezug auf Hilfsmittel bestehen - wie in authentischen professionellen Übersetzungssituationen - keine Beschränkungen.

Beim Unterricht im 4. Semester geht es also um die Lösung von kommunikativen Aufgaben, genauer um die Gestaltung des Zieltextes im Rahmen eines Übersetzungsauftrages. Zentral ist deshalb nicht der Ausgangstext an sich, sondern wie dieser für den spezifischen Textproduktionsauftrag für den Zieltext zu gestalten ist. Der Unterricht für den hier untersuchten Jahrgang lässt sich wie folgt einteilen:

Im Theorieteil wurde zusätzlich zu einer Einführung in die Textanalyse mit Übersetzungstheorie gearbeitet. Den Schwerpunkt bildeten dabei das Loyalitätskonzept von Christiane Nord sowie ihre Typologisierung in dokumentarisches und instrumentelles Übersetzen (Nord 1997). Lernziel für diese Unterrichtseinheit ist die Breite des Übersetzungsbegriffs gewesen: einerseits das Verstehen von Übersetzen als Text sowie andererseits, dass ein Text in verschiedene Richtungen - je nach Übersetzungsauftrag - übersetzt werden kann.

Im Strategieteil wurden konkrete Übersetzungsfälle in bezug auf die Lösung von Übersetzungsaufträgen analysiert. Zentral war dabei, wie der Ausgangstext für die Gestaltung eines funktionsgerechten Zieltextes umgestaltet wurde: nicht nur ,rein sprachlich', sondern auch nach der Relevanz von Inhaltselementen, nach allgemeinen Argumentationsmustern, nach spezifischen Textsortenkonventionen usw. Gerade im Strategieteil, wo also authentische Übersetzungsaufträge analysiert wurden, wurde mit translatorischer Relevanz durch die didaktisch gemeinte Formel, ,wie gehe ich im Rahmen dieses konkreten Auftrags mit dem Ausgangstext um?' explizit gearbeitet.

Im Textproduktionsteil haben die Studierenden alle zwei Wochen Klausuren abgegeben, die dann korrigiert wurden. Diese Arbeiten wurden alle zu zweit oder zu dritt geschrieben und enthielten ab Mitte des Semesters konkrete Fragen zum auftragsbedingten Umgang mit dem Ausgangstext.

19 Zusätzlich zu den Übersetzungsaufträgen werden Fragen zur Übersetzungsstrategie sowie zur Textanalyse gestellt. 
Durch das Lernen von einem differenzierten Übersetzungsbegriff und damit zusammenhängenden Analysekategorien sowie vor allem durch die Analyse von authentischen Übersetzungsaufträgen und die eigene, in Gruppen durchgeführte Lösung von und Reflektion über Übersetzungsaufträge ist die Arbeit mit translatorischer Relevanz im Unterrichtsprogramm sowohl theoretisch wie auch praktisch-strategisch eingebettet.

\subsection{Fragestellung und Methode}

In der vorliegenden Untersuchung wurde im Rahmen der Prüfungsklausur im 4. Semester konkret nach Art und Stellenwert von Übersetzungsproblemen gefragt: „Erläutern Sie die wichtigsten Probleme, die Sie bei der Übersetzung dieses Textes hatten und begründen Sie an selbstgewählten Beispielen Ihre Lösungsvorschläge“ 20 . Nun ist ja die Frage nach der unterschiedlichen Relevanz von Merkmalen des Ausgangstextes für die Zieltextproduktion bei jedem Übersetzungsauftrag grundsätzlich zu stellen. Bei den hier untersuchten Übersetzungsaufträgen wurde außerdem bei der Übersetzung vom Deutschen ins Norwegische im Auftrag auch konkret nach der unterschiedlichen Relevanz von Ausgangstextinhalten für den Zieltext gefragt: Bei der Übersetzung des Textes Immer mehr Menschen in der Schuldenfalle sei ,auf das Problem dieser besonderen Verschuldung zu achten und etwas weniger auf Details, die nur für deutsche Leser von Relevanz wären“21. Die Anzahl der Studierenden - nur 9 - ist natürlich keine besonders gute Grundlage für Generalisierungen. Aber als empirische Grundlage für Arbeitshypothesen dürfte die vorliegende Materialgrundlage einen Wert haben, vor allem zusammengehalten mit Ergebnissen von einer Arbeit mit einer vergleichbaren Problemstellung (= Kvam 1998).

Die Antworten der Studierenden wurden nach folgendem Muster evaluiert: Inwieweit haben die Studierenden Fragen der translatorischen Relevanz als Übersetzungsproblem aufgeführt und wie haben sie solche

\footnotetext{
20 Aufgabe B 2 bei der Prüfung „Fagspråklig tekstproduksjon“ am 6. - 7. Mai 2003 am Fachbereich Fremdsprachen und Sozialwissenschaften der Gesamthochschule Østfold in Halden/Norwegen.

21 Dieser Text aus der Internetausgabe der Welt am Sonntag den 24.4.2003 war für die Osloer Zeitung Aftenposten zu übersetzen.
} 
Fragen mit welchem Erfolg bzw. welchem Ergebnis zu lösen versucht. Als Maßstab für die Evaluation wurde die Benotung bei der Prüfung benutzt22.

\subsection{Ergebnisse}

Bei der Übersetzung ins Deutsche dominierten bei allen neun Studierenden lexikalische Probleme, sprich: Vokabelprobleme die Problemanalyse der Studierenden. Sechs Studierende haben ausschließlich lexikalische Fragen als Probleme erwähnt. Die Übersetzungsstrategie von diesen sechs Studierenden erwies sich auch als sehr ausgangstexttreu: Es wurde Satz für Satz übersetzt, von einem textsortengerechten Stil des Zieltexts war nicht die Rede, dagegen von relativ soliden grammatischen und lexikalischen Fehlern. Die Noten waren im Bereich C - F. Drei der neun Studierenden hatten jedoch auch Fragen der translatorischen Relevanz berücksichtigt. Eine hat dies als wichtiges Übersetzungsproblem explizit erwähnt und das Problem in ihrer Übersetzung auch relativ gut bewältigt, zwei weitere Studierende haben solche Fragen zwar nicht als Übersetzungsproblem explizit erwähnt, aber in ihren Übersetzungen Relevanzentscheidungen systematisch getroffen und diese Probleme auch weitgehend erfolgreich gelöst. Die Noten waren im Bereich A - B.

Bei der Übersetzung ins Norwegische waren die Ergebnisse noch deutlicher. Hier wurde, wie in 3.2. erwähnt, im Übersetzungsauftrag auch explizit nach der unterschiedlichen Relevanzsetzung von Ausgangstextinhalten gefragt. Auch hier wurden lexikalische Probleme hoch eingestuft. Auch hier lassen sich die Antworten der Studierenden in zwei Gruppen einteilen, und zwar genau wie bei der Übersetzung ins Deutsche in sechs teils sehr schlechte und drei teils sehr gute Leistungen. Bei der erstgenannten Gruppe wurden lexikalische Fragen vor allem die Frage nach der Übersetzung deutscher Institutionen ins Norwegische 23 - als wichtigstes, oft alleiniges Übersetzungsproblem erwähnt. Zwei von diesen sechs Studierenden erwähnten zusätzlich zu

22 Im norwegischen Hochschulsystem wird jetzt ein Notensystem bestehend aus 6 Noten $(\mathrm{A}-\mathrm{F})$ eingeführt. A ist die beste Note, $\mathrm{F}$ ist durchgefallen. Für den Notenbereich ,bestanden' stehen also 5 Noten $(\mathrm{A}-\mathrm{E})$ zur Verfügung.

23 Beispiele solcher Institutionen waren das Deutsche Institut für Wirtschaftsforschung in Berlin sowie der Bundesverband der Verbraucherzentralen in Berlin. 
den Vokabelproblemen eine unterschiedliche Relevanzsetzung von referentiellen Elementen des Ausgangstextes im Zieltext, allerdings ohne sich mit diesem Problem in ihren Übersetzungen zu beschäftigen: Bei allen diesen sechs Studierenden waren die Zieltexte Satz für Satz und sehr ausgangstextnah übersetzt und darüber hinaus in einem sehr deutsch klingenden, sprich: relativ extrem nominalisierten Norwegisch geschrieben.

Bei der zweitgenannten Gruppe, also bei den Studierenden mit den recht guten Leistungen, hat eine Studentin zwar sklavisch die thematische Progression im Ausgangstext wiedergegeben und dadurch Fragen der unterschiedlichen Relevanz von Ausgangstextinhalten für den Zieltext nicht berücksichtigt, allerdings einen textsortengerechten und stilistisch schönen norwegischen Zieltext geschrieben und dadurch gerade eine B gerettet. Zwei der neun Studierenden haben unter den Übersetzungsproblemen Fragen der translatorischen Relevanz den höchsten Stellenwert gegeben. Die Übersetzungsstrategie entsprach auch deutlich dieser Problemeinschätzung. Die Zieltexte waren mit dem Übersetzungsauftrag kompatibel und in einem textsortengerechten, nicht verdeutschten Norwegisch geschrieben. Diese beiden Arbeiten wurden mit der Note A beurteilt.

\section{Schlussfolgerung und offene Fragen}

Es ist, wie erwähnt, nicht möglich, auf der Basis dieser Datenmenge weitreichende Schlussfolgerungen zu ziehen. Eine mögliche Tendenz in der vorliegenden Untersuchung ist trotzdem erwähnenswert: Bei einer Fokussierung auf lexikalische Probleme werden Fragen der Relevanz von anderen textuellen Elementen, wie in diesem Übersetzungsfall vor allem die auftragsspezifische Bearbeitung von referentiellen Elementen im Ausgangstext für den Zieltext, kaum oder gar nicht berücksichtigt. Eine solche vokabelorientierte Übersetzung wird auch oft von einer kopierenden Übersetzungsstrategie begleitet: Maßstab für den Zieltext ist die Wort- und Satzabfolge im Ausgangstext. Dies betrifft in den hier untersuchten Übersetzungen nicht nur die Übersetzung in die Fremdsprache Deutsch, sondern auch diejenige in die Muttersprache Norwegisch.

Dieses Ergebnis stimmt auch mit der vokabelorientierten und strukturkopierenden Übersetzungsstrategie, die auf der Grundlage von 14 
Studierenden in Kvam 1998 nachgewiesen wurde: Studierende, die die etymologisch nächstliegende Variante eines Lexems im Ausgangstext gewählt haben, hingen an dieser Wahl sowie an einer ausgangstextkopierenden Übersetzungsstrategie fest (ebd., 290f.). Trotz der unterschiedlichen Problemstellungen - eine Analyse von translatorischer Interferenz 24 in Kvam 1998 und die Analyse von translatorischer Relevanz in der vorliegenden Arbeit - scheint es zwischen einer grundsätzlichen Vokabelorientiertheit bei der Problemeinschätzung des Übersetzers und einer ausgangstextkopierenden und dadurch oft auftragsabgewandten Übersetzungsstrategie einen Zusammenhang zu geben.

Umgekehrt lassen sich bei den Studierenden, die Fragen der translatorischen Relevanz als wichtigen Problembereich aufgeführt hatten, auftragsorientierte und weitgehend gute Übersetzungen nachweisen. Aber auch durch die magere empirische Grundlage von neun Studierenden kommt zum Ausdruck, dass die These wenn lexikalische Fokussierung, keine Relevanzsetzungen und dadurch schlechte Übersetzungen; wenn Relevanzsetzungen, dann auch gute Übersetzungen zu weit gefasst ist. Denn zwei von den Studierenden hatten Relevanzfragen bei der Übersetzung ins Deutsche nicht erwähnt, dafür eben lexikalische Probleme. Nichtsdestotrotz waren ihre Übersetzungen gut (A und B), und zwar gerade in bezug auf Relevanzfragen. Diese Studierenden verfügten über einen guten Wortschatz und eine gute grammatische Korrektheit in der Fremdsprache Deutsch, sie hatten auch einen textsortengerechten Stil in ihren norwegischen Zieltexten und waren auch bei der Lösung der in der Prüfung gestellten textlinguistischen Fragen deutlich besser als die anderen Studierenden. Ihre sprachlichen Fertigkeiten, ihre strategisch-analytische Kompetenz und ihr theoretisches Grundwissen waren also den anderen Studierenden deutlich überlegen. Die Voraussetzungen für eine text- und auftragsorientierte Analyse und eine davon abgeleitete konsistente Übersetzungsstrategie waren also bei diesen beiden deutlich besser als bei den anderen.

Dies deutet darauf hin, dass eine lexikalische Problemfokussierung nicht notwendigerweise einer ausgangstextkopierenden, Relevanz-

24 Vgl. hierzu Näheres in Kvam 1998, 284ff.. 
fragen nicht berücksichtigenden Übersetzungsstrategie entspricht. Es kommt eher darauf an, in welchem Rahmen lexikalische Probleme aufgegriffen werden: Zwar werden bei Studierenden mit schlechter textanalytischer Kompetenz lexikalische Elemente als unmittelbare Probleme in Angriff genommen. Sie werden weitgehend als isolierte Probleme im Rahmen eines grundsätzlich ausgangstextkopierenden und auftragsabgewandten Übersetzungsverfahrens gelöst. Lexikalische Übersetzungsprobleme werden jedoch auch von Studierenden mit guter textanalytischer Kompetenz aufgegriffen und als zentral eingestuft. Solche Probleme werden bei dieser Gruppe von Studierenden jedoch nicht als isolierte Vokabelprobleme, sondern als im Rahmen einer text- und auftragsorientierten Übersetzungsstrategie betrachtet. Im Rahmen einer solchen Übersetzungsstrategie kann sehr wohl auf den Stellenwert bzw. die Relevanz von lexikalischen Elementen im Ausgangstext für den Zieltext eingegangen werden - z. B., ob oder wie differenziert sie im Zieltext zu realisieren sind.

Die Analyseergebnisse der vorliegenden Untersuchung machen deutlich, dass es einen Zusammenhang zwischen einer auftragsorientierten Übersetzungsstrategie und der bewussten Verwendung von translatorischer Relevanz als Analysewerkzeug für die Analyse des Ausgangstextes zu geben scheint. Dieses sehr generelle Ergebnis über einen Zusammenhang zwischen Kompetenzvoraussetzungen und einer spezifischen Wahl von Übersetzungsstrategien ist zwar interessant, aber in der vorliegenden Untersuchung sehr dünn empirisch untermauert. Hier sind weitere Analysen auf breiter empirischer Grundlage nötig, bei denen vor allem das Verhältnis zwischen textlinguistischem Grundwissen und textanalytischer Kompetenz einerseits und übersetzungsstrategischer Reflexion und übersetzerischer Praxis andererseits systematisch zu untersuchen wäre.

\section{Literaturverzeichnis}

Adamzik, Kirsten 2000: Was ist pragmatisch orientierte Textsortenforschung? In Adamzik, Kirsten (Hg.): Textsorten. Reflexionen und Analysen (= Textsorten 1). Tübingen: Stauffenburg, $91-112$.

Auer, Peter 1999: Sprachliche Interaktion. Eine Einführung anhand von 22 Klassikern (= Konzepte der Sprach- und Literaturwissenschaft 60). Tübingen: Niemeyer. 
Berger, Klaus/Nord, Christiane 2000: Verstandene Fremdheit: Ein neuer Skopos für alte Texte. In Kadric, Mira et al. (Hg.): Translationswissenschaft (= Festschrift für Mary Snell-Hornby zum 60. Geburtstag). Tübingen: Stauffenburg, 213 - 225.

Brinker, Klaus 2002: Textsortenbeschreibung auf handlungstheoretischer Grundlage (am Beispiel eines Erpresserbriefs). In Adamzik, Kirsten (Hg.): Texte - Diskurse - Interaktionsrollen. Analysen zur Kommunikation im öffentlichen Raum (= Textsorten 6). Tübingen: Stauffenburg, $41-59$.

Ehlich, Konrad 1996: Funktion und Struktur schriftlicher Kommunikation. In Steger, Hugo/Wiegand, Herbert Ernst (Hg.): Schrift und Schriftlichkeit (= Handbücher zur Sprach- und Kommunikationswissenschaft 10.1). Berlin, New York: de Gruyter, 18 -41 .

Engberg, Jan 2002: Warum auch bei Fach-Übersetzung Interpretation notwendig ist - und worauf sie gestützt werden kann. In Albert, Marina Foschi/Hepp, Marianne (Hg.): Germanistische Linguistik und Spracherwerb an den italienischen Universitäten (=Jacques e i suoi quaderni 39). Pisa: Universität Pisa, 157 - 168.

Halliday, Michael A. K. 1985: An Introduction to Functional Grammar. London etc.: Edward Arnold.

Heinemann, Margot/Heinemann, Wolfgang 2002: Grundlagen der Textlinguistik. Interaktion - Text - Diskurs (= Reihe Germanistische Linguistik 230). Tübingen: Niemeyer.

Klein, Wolfgang 1991: Was kann sich die Übersetzungswissenschaft von der Linguistik erwarten? In Zeitschrift für Literaturwissenschaft und Linguistik 21. Göttingen: Vandenhoeck\&Ruprecht, $104-123$.

Kvam, Sigmund 1998: Strukturelle Interferenzen als translatorisches Problem am Beispiel norwegisch-deutscher fachsprachlicher Übersetzungsfälle. In Pors, Harald et al. (Hg.): Sprachgermanistik in Skandinavien III (= Akten des IV. Nordischen Germanistentreffens). Aarhus: Handelshøjskolen i Aarhus, 283 - 300.

Kvam, Sigmund 2002: Kontraktive Konstruktionen als Textgestaltungsmittel. Eine Fallstudie am Beispiel eines deutsch-norwegischen fachsprachlichen Paralleltextes (= Hermes skriftserie 3). Aarhus: Handelshøjskolen i Aarhus.

Nord, Christiane 1997: Translation as a Purposeful Activity (= Translations Theories Explained 1). Manchester: St. Jerome.

Nothdurft, Werner/Reitemeier, Ulrich/Schröder, Peter 1994: Beratungsgespräche. Analyse asymmetrischer Dialoge (= Forschungsberichte des Instituts für deutsche Sprache 61). Tübingen: Narr.

Ortner, Hanspeter/Sitta, Horst 2002: Was ist der Gegenstand der Sprachwissenschaft? In Linke, Angelika et al. (Hg.): Sprache und mehr. Ansichten einer Linguistik der sprachlichen Praxis (= Reihe Germanistische Linguistik 245). Tübingen: Niemeyer, $1-31$. 
Prunc, Erich 1997: Versuch einer Skopostypologie. In: Grbic, Nadja/Wolf, Michaela (Hg.): Text - Kultur - Kommunikation. Translation als Forschungsaufgabe (= Studien zur Translation 4). Tübingen: Stauffenburg, 33 - 52.

Reiß, Katharina 2000: Adäquatheit und Äquivalenz als Schlüsselbegriffe der Übersetzungstheorie und -praxis. In Snell-Hornby, Mary/Kadric, Mira (Hg.): Grundfragen der Übersetzungswissenschaft. Wiener Vorlesungen (= WUV Studienbücher Geisteswissenschaften 1). Wien: WUV-Universitätsverlag, 106 - 129.

Solfjeld, Kåre 2000: Sententialität, Nominalität und Übersetzung. Eine empirische Untersuchung deutscher Sachprosatexte und ihrer norwegischen Übersetzungen (= Osloer Beiträge zur Germanistik 26). Frankfurt am Main etc.: Peter Lang.

Tirkkonen-Condit, Sonja 1985: Argumentative Text Structure and Translation (= Studia Philologica Jyväskyläensia 18). Jyväskylä: Universität Jyväskylä. 